A simpler and less expensive approach to platelet counting with improved precision using the Technicon Autocounter.

\author{
M. M. LAVER AND M. F. HOWELl Department of \\ Clinical Chemistry and Department of Clinical \\ Haematology, Essex County Hospital, Colchester, \\ Essex, UK
}

The Technicon Platelet Autocounter is a fully automated system for counting platelets rapidly using whole blood without prior manipulation. An evaluation of the instrument has already been documented (Rowan et al., 1972). The introduction of the Technicon Autocounter in this laboratory produced several problems which apparently are not unique to us. This paper explains these problems and how they were overcome.

As new users of the Technicon Autocounter we had some adverse initial reactions to (a) the use of a complex manifold for a relatively simple estimation; (b) the large amount of urea reagent used, of which $20 \%$ is pumped to waste; and (c) the large volume of sample required.

To overcome these initial problems a new manifold was designed (Fig. 1), which overcame these defects and is constructed very simply in comparison with the original manifold. Using the modified manifold a $73 \%$ reduction in sample aspiration and a $33 \%$ reduction in urea usage was achieved.

Having successfully introduced the new manifold, investigations had still to be carried out on two further problems, namely, poor peaks and poor operation of the wash valve.

\section{Poor peaks}

We encountered difficulty in obtaining smooth peaks on whole blood, especially in those patients with elevation of the erythrocyte sedimentation rate and in those with a disturbance of immunoglobulins.

This was investigated by doing manual counts using an Improved Neubauer counting chamber on consecutive fractions of the same sample at the point of entry to the Autocounter. The results obtained confirmed the findings of Rowan et al., (1972), who reported that the greater proportion of particles is found in the latter end of the sample. This we assumed to be a contributory factor to

Received for publication 12 September 1977 poor peaking. Technicon's recommended procedure to improve peak characteristics is to inject air bubbles into the sample at the top of the sample probe by means of a PT2 fitting. This procedure had only very limited success, and we modified it by injecting urea solution (Fig. 2) via pump tube 17 into the PT2 fitting at the top of the probe. The recorder tracings were immediately improved. We obtained further improvements by placing an extra coil in the primary dilution system, and by introducing the sample and air into the urea solution by means of an $\mathrm{H} 3$ fitting inserted after the $\mathrm{HO}$ fitting coming from the pump (Fig. 1).

\section{Poor operation of wash-valve}

We investigated the flow characteristics of the Autocounter to devise a way to eliminate the washvalve. This has the innate (and infuriating!) habit of both leaking and blocking, which resulted in poor or no peaks.

On the Autoanalyser systems in the Department of Clinical Chemistry the wash characteristics are dependent on the ratio of sample to water aspirated, which can be varied by the use of cams supplied by Technicon Instruments Ltd. As a wash-valve is not found to be necessary on those systems we suspended the use of the wash-valve on the Autocounter and made new connections to the debubbler, as shown in Fig. 3. By trying different cams and measuring the carry-over (Broughton et al., 1969) a sample/ wash ratio of $2: 1$ and a sampling rate of 60 per hour were achieved and found to be satisfactory; the carry-over expressed as percentage interaction (Young and Gochman, 1972) was 2\%, compared with a figure of $5 \%$ by the original method. The increased sampling rate of 60 samples per hour provided a further saving in the amount of urea reagent used per sample, increasing the total saving to $55 \%$.

The linearity of the modified system was assessed and found to be satisfactory (Fig. 4) by diluting platelet-enriched plasma with platelet-free plasma, thus producing a range of platelet concentrations up to $725 \times 10^{9} / 1$, using the Technicon platelet reference reagent to calibrate the Autocounter.

The modifications to the manifold result in the volume of sample aspirated being reduced to $120 \mu 1$. This enables small samples to be directly estimated and generally eliminates the need for predilution and aspiration through the secondary dilution system, as suggested by Technicon Ltd.

The modifications to the Technicon Platelet Autocounter, as described above, have been in routine use for one year and have yielded a fast, accurate, and reliable method for the estimation 


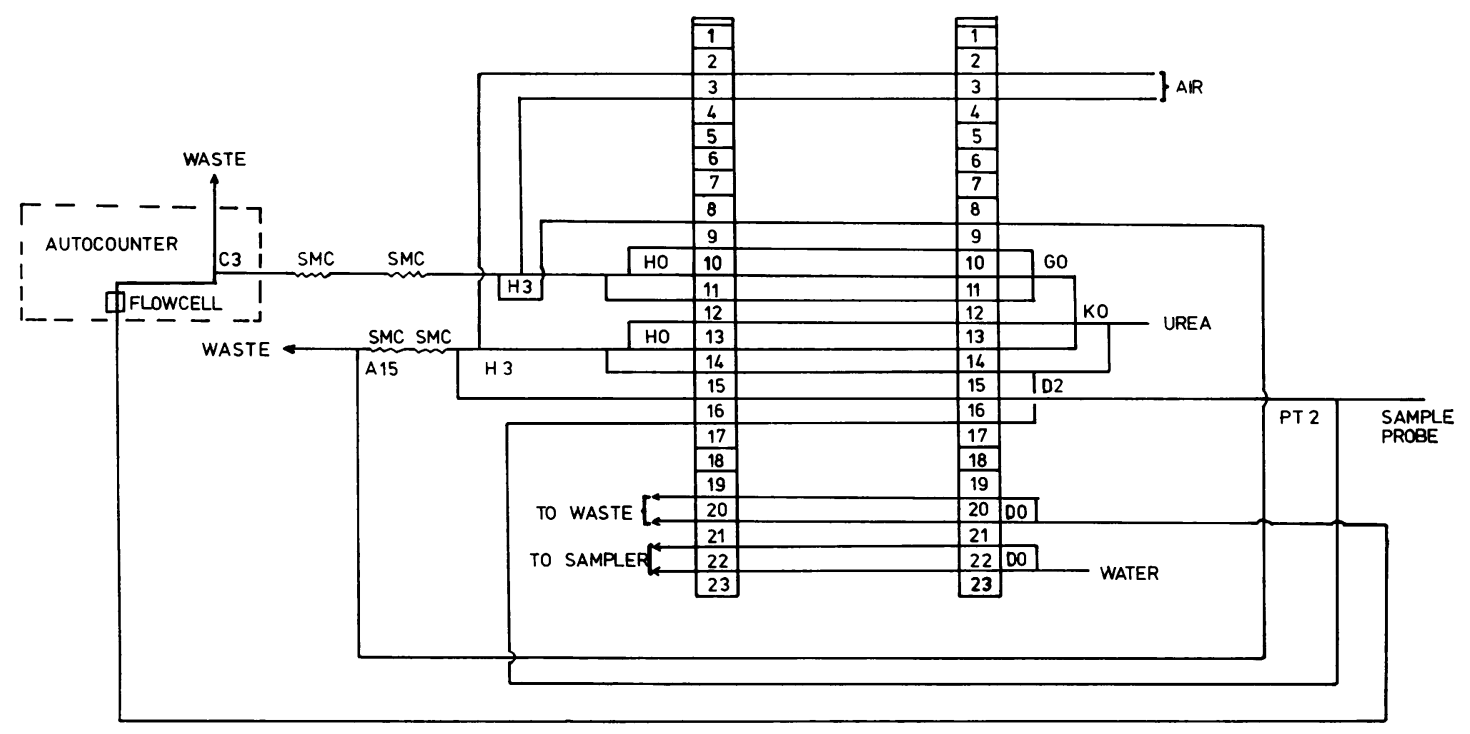

SMC - SINGLE MIXING COIL

\begin{tabular}{cl}
\hline Pump tube size & Tube int. diam. (in). \\
\hline No. 3 & 0.040 \\
4 & 0.040 \\
9 & 0.015 \\
10 & 0.060 \\
11 & 0.060 \\
12 & 0.060 \\
13 & 0.065 \\
14 & 0.065 \\
15 & 0.065 \\
16 & 0.030 \\
17 & 0.020 \\
20 & 0.073 \\
21 & 0.073 \\
22 & 0.073 \\
23 & 0.090 \\
\hline
\end{tabular}

Fig. 1 Modified platelet manifold.

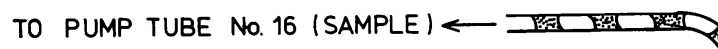

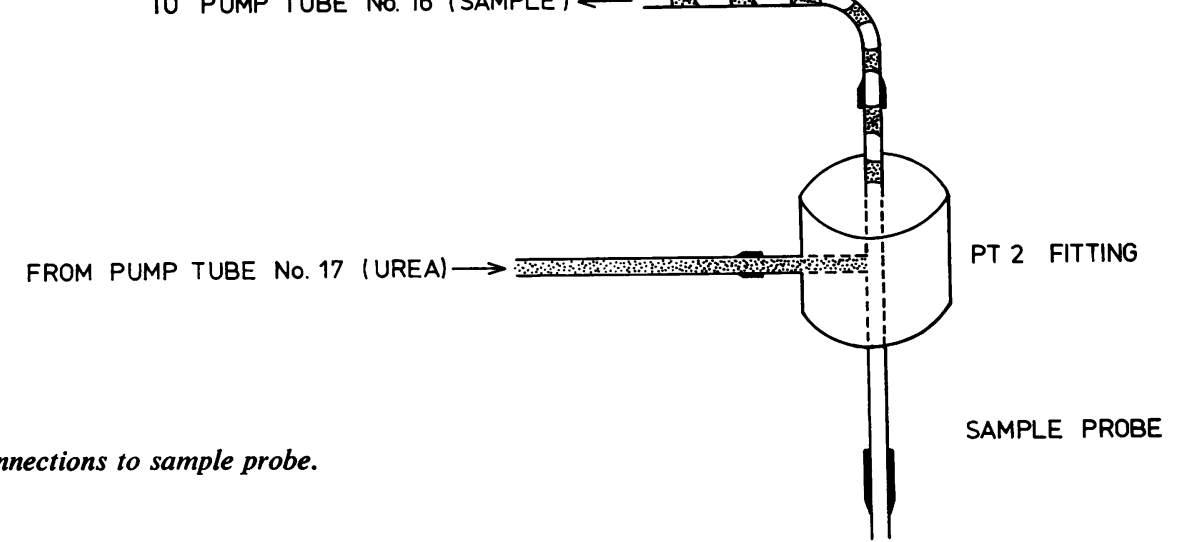

Fig. 2 Diagram of connections to sample probe. 


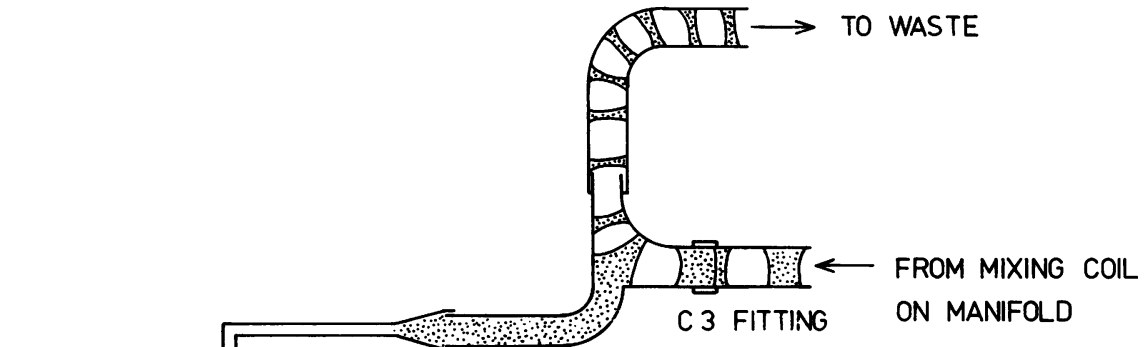

Fig. 3 Diagram of flow connections to flow-cell.

TO PUMP [PULL THROUGH]

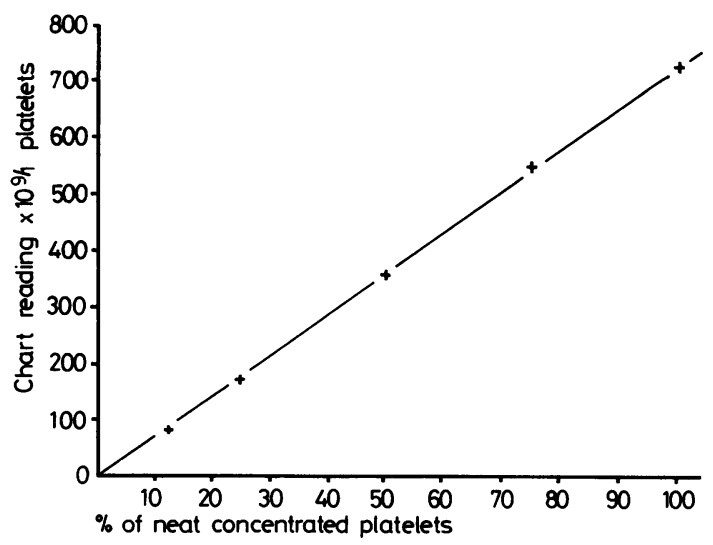

Fig. 4 Standard curve to assess linearity using plateletenriched plasma diluted with platelet-free plasma.

of platelets. The running costs for reagents and consumables have been reduced by at least $50 \%$, and the performance of the instrument has been greatly enhanced, resulting in a system which is more acceptable to the user.

We acknowledge the technical assistance of Mrs. S. D. Giddings. We also thank Mrs. G. A. Knox, who typed the manuscript.

\section{References}

Broughton, P. M. G., Buttolph, M. A., Gowenlock, A. H., Neil, D. W., and Skentelbery, R. G. (1969). Recommended scheme for the evaluation of instruments for automatic analysis in the clinical biochemistry laboratory. Journal of Clinical Pathology, 22, 278-284. Rowan, R. M., Allan, W., and Prescott, R. J. (1972). Evaluation of an automatic platelet counting system utilizing whole blood. Journal of Clinical Pathology, 25, 218-226.

Young, D. S., and Gochman, N. (1972). Methods for assuring quality data from continuous flow analysis. Standard Methods of Clinical Chemistry, 7, 293-310. 